\title{
Post-traumatic stress disorder and head injury as a dual diagnosis: "islands" of memory as a mechanism
}

\author{
Nigel S King
}

\begin{abstract}
This case study describes post-traumatic stress disorder (PTSD) and head injury after a road traffic accident involving a pedestrian. Previous studies have proposed two mechanisms by which this dual diagnosis may occur: (1) when post-traumatic amnesia and retrograde amnesia are small or non-existent and (2) when non-declarative memory systems for the traumatic event are in operation. This case study demonstrates a third mechanism-"islands" of memory within posttraumatic amnesia.
\end{abstract}

$(\mathcal{F}$ Neurol Neurosurg Psychiatry 1997;62:82-84)

Keywords: post-traumatic stress disorder; head injury; "islands" of memory

It is clear that circumstances in which a person sustains a head injury (for example, assaults, road traffic accidents, industrial accidents) may often be those where "threatened death or serious injury" (DSM IV) ${ }^{1}$ also occurs ${ }^{2}$ and therefore where post-traumatic stress disorder (PTSD) might also be expected. The dual diagnosis of PTSD and head injury, however, is uncommon, particularly if judged by the number of published examples of its occurrence. ${ }^{3-6}$ This is probably due to several factors. Firstly, organic amnesia which commonly accompanies head injury (in the form of unconsciousness, post-traumatic amnesia, or retrograde amnesia), is thought to protect most patients with head injury from developing PTSD. Indeed, some writers have used rigid definitions of PTSD and head injury to argue that "PTSD and MTBI (mild traumatic brain injury) are mutually incompatible since patients who sustain PTSD simply cannot 'forget' the traumatic event, whereas patients who sustain MTBI (for example, cerebral concussion) have no recollection of the traumatic event."7

A second factor can be the existence of Department, Department

Rehabilitation Centre, Abingdon Road Oxford OX1 4XD, UK N S King

Received 15 April 1996 and in revised form 17 July 1996 Accepted 13 August 1996
A third factor is the interpretation of any amnesia for the event. A lack of full memory for the traumatic event can be due to organic amnesia and thus a sign of head injury or due to psychogenic amnesia and thus a potential PTSD symptom of avoidance. No agreed method of distinguishing between these types of amnesia has been reported. ${ }^{5}$ It seems reasonable, however, that if psychogenic amnesia in PTSD is primarily an avoidance or dissociative reaction then it is more likely to occur for aspects of the event which are perceived as being the most traumatic rather than for those that are perceived as less so. It is no doubt possible, however, for an investigator to attribute amnesic aspects of post-traumatic amnesia to PTSD or vice versa.

Two mechanisms by which both a head injury and PTSD can occur have been highlighted:

(1) when retrograde amnesia or post-traumatic amnesia is very small or non-existent. In such cases memories of a traumatic incident immediately before or immediately after the head injury may be present. ${ }^{7}$

(2) Contemporary memory theory posits the existence of multiple and independent memory systems. These include the declarative or explicit system (stored experience actually or potentially accessible to conscious recollection) and the non-declarative or implicit system (stored experience not accessible to consciousness). ${ }^{8}$ Within such theories the reexperiencing of a traumatic event may be via "intense psychological distress on exposure to internal or external cues that symbolise or resemble an aspect of the traumatic event" (DSM IV) without conscious recall of the event itself. $^{5}$

This paper presents a case study which highlights a third mechanism by which head injury and PTSD can co-occur- "islands" of memory during post-traumatic amnesia. Posttraumatic amnesia is usually defined as the period of time between receiving a head injury and the resumption of normal continuous memory.9-11 "Islands" of memory or recollections of events which occur outside continuous memory for events are a common feature of post-traumatic amnesia ${ }^{10} 12$ and occur in at least a third of all cases of mild head injury. ${ }^{13}$ In Gronwall and Wrightson's sample "islands" of memory were usually reported for specific 
changes in events-for example, being helped into an ambulance, being discharged from hospital etc. Forty four per cent of those who reported "islands" had them for events less than five minutes after injury and $71 \%$ reported them for events less than 15 minutes after injury.

\section{Case report}

A 21 year old unemployed man sustained a head injury as the result of being hit by a car from behind as a pedestrian while hitch hiking home at night. $\mathrm{He}$ also had considerable orthopaedic injuries. He had no previous history of head injury, psychiatric treatment, or psychological trauma, but reported being an occasional cannabis user.

Evidence for head injury included the following:

(1) Ambulance records which stated that (a) he was "probably knocked out for a few minutes according to witness"; $(b)$ he was struck at about $40 \mathrm{mph}$ and was thrown on to the bonnet of the car, smashing the windshield (almost certainly with his head); (c) he had a wound to the front of his head and significant pain at the back of the head; and (d) he had neck injuries that required a collar.

(2) Hospital records on admission diagnosed (a) mild head injury and recommended hourly neurological observations; (b) boggy haematoma over the left temporoparietal area; $(c)$ left frontal skull laceration; and (d) laceration to the chin

(3) Medical discharge summary included the diagnosis of head injury.

(4) At a routine follow up nine days after injury post-traumatic amnesia was established by retrospective questioning. He described a single memory immediately after his head injury, of lying in the road, seeing the car that had hit him turn around, and him having great difficulties in pulling himself to the side of the road due to his orthopaedic injuries. The intensely traumatic aspect of this memory was the mistaken perception that the car had intentionally hit him and that it had turned round to "finish him off". He had no other memories until $2 \frac{1}{2}$ days later on the trauma ward in hospital. Normal memory resumed almost immediately after this time. Post-traumatic amnesia was thus assessed as $2 \frac{1}{2}$ days.

(5) At the follow up interview he reported pronounced postconcussion symptoms, which included sensitivity to light, headaches, concentration difficulties, forgetfulness, dizziness, sleep disturbance, irritability, depression, feelings of frustration, and restlessness. He also reported post-traumatic stress symptoms.

At an interview four months after the injury almost all of his symptoms were worse and he fulfilled DSM IV criteria for PTSD even if the symptoms of severe concentration difficulties, sleep disturbance, and irritability were arbitrarily attributed to postconcussion symptoms rather than PTSD. Symptoms of PTSD included automatic thoughts of the "island" of memory of the accident many times a day, nightmares five to seven nights a week of the "island" of memory, sweaty palms, and significantly increased heart rate and arousal levels when near most roads, phobic avoidance of all but minor roads, persistent efforts to avoid thoughts and conversations about the accident, very diminished participation in activities (for example, staying in his bedroom for up to three days consecutively), feelings of detachment from others (particularly family members), sleeping for only about $25 \%$ of the time before the accident, irritability and verbal outbursts of anger, difficulties concentrating as highlighted by being able to read only one or two pages of a book before losing concentration (compared with reading a book every few days before the injury), exaggerated startle response, hypersensitivity to most potential dangers inside and outside his house, and impairment in social functioning (in the form of drinking to excess, much diminished interest in socialising with friends, and phobic avoidance of busy roads).

The criteria for fulfilling the diagnosis of PTSD was confirmed by scores on the PTSD symptom scale. ${ }^{14}$

\section{Discussion}

This case study presents someone with both a head injury and PTSD arising from the same event. A key feature of the PTSD was the reexperiencing of intrusive images, thoughts, and nightmares of an "island" of memory during post-traumatic amnesia after the head injury. The case therefore infers a third mechanism by which the dual diagnosis of head injury and PTSD may occur. An "island" of memory for part of the traumatic event can be responsible for the recurrent intrusive recollections of the trauma which are a key feature of the diagnosis of PTSD (DSM IV). This mechanism is consistent with previous explanations of "islands" of memory which consider them to happen only when a strong "stimulus" occurs during partial recovery from a head injury. ${ }^{1315}$ Gronwall and Wrightson ${ }^{13}$ postulated that the process of recovery after concussion may occur in two phases. The initial phase often occurs during the first hour after mild head injury and the second phase then follows. They argue that "islands" of memory usually occur during the first phase and only "when a stimulating event coincides with a peak arousal, providing enough external activation to push the peak above the threshold level for memory". ${ }^{13}$ An explanation of the patient's "island" of memory is thus that the notable heightened state of arousal present immediately after his injury was sufficient to generate declarative memory of the event which would not have been possible due to post-traumatic amnesia under less traumatic circumstances. This memory then became a key mechanism by which PTSD developed.

Possible alternative mechanisms for "islands" of memory in cases such as this patient are (1) a false memory (based on what the patient is told after the event) being inserted into the period of post-traumatic amnesia and (2) an isolated memory being 
retained while recall of the surrounding events is lost due to psychogenic processes. The first is unlikely in the present patient because the car actually came back to help him and he was consistently told this. Any potential false memory would therefore have been of this rather than his more traumatic perception of the event. The second is unlikely because his "island" is of probably the most traumatic aspect of the accident, which is precisely the part most likely to be lost if psychogenic mechanisms were in operation.

Unfortunately, the treatment implications are not clarified by the case report as treatment was declined. It is hoped, however, that an awareness of this mechanism will allow clinicians who are treating such patients to develop appropriate methods of intervention.

Thanks are due to Dr DT Wade and Mrs J McGrath for helpful comments and to Mrs A White for typing this manuscript. Thanks also to the Department of Health for funding the Oxford Head Injury Service as part of their traumatic brain injury initiative.

American Psychiatric Association. Diagnostic and statistical manual of mental disorders. 4th ed (DSM IV). Washington, DC: APA, 1994.
2 Middelboe T, Andersen HS, Birket-Smith M, Friis ML Minor head injury: impact on general health after one year. A prospective follow-up study. Acta Neurol Scand year. A prosp

3 McMillan TM. Post-traumatic stress disorder and severe head injury. Br f Psychiatry 1991;159:431-3.

4 Horton AH. Post-traumatic stress disorder and mild head trauma: follow-up of a case study. Percept Mot Skills 1993;76:243-6.

5 Layton BS, Wardi-Zonna K. Post-traumatic stress disorde with neurogenic amnesia for the traumatic event. The Clinical Neuropsychologist 1995;9:2-10.

6 McGrath J. Cognitive impairment associated with post traumatic stress disorder and minor head injury: case report Neuropsychological Rehabilitation 1997 (in press).

7 Sbordone RI, Liter JC. Mild traumatic brain injury does not produce post-traumatic stress disorder. Brain Inj 1995;9:405-12.

8 Squire CR. Declarative and nondeclarative memory: multiple brain systems supporting learning and memory. fournal of Cognitive Neuroscience 1992;4:232-43.

9 Russell WR, Smith A. Post traumatic amnesia after closed head injury. Arch Neurol 1961;5:16-29.

10 Whitty CWM, Zangwill OL. Amnesia. 2nd ed. London Butterworths, 1997:118-35.

11 Lezak MD. Neuropsychological assessment. New York Oxford University Press, 1983:165-75.

12 Forrester G, Encel J, Geffen G. Measuring post-traumatic amnesia (PTA): an historical review. Brain Inj 1994;8: 175-84.

13 Gronwall $D$, Wrightson $P$. Duration of post-traumatic amnesia after mild head injury. Fournal of Clinical amnesia after mild head inju

14 Foa EB, Riggs DS, Dancu CV, Rothbaun BO. Reliability and validity of a brief instrument for assessing postand validity of a brief instrument for assessing posttraumatic

15 Russell WR, Nathan PW. Traumatic amnesia. Brain 1946, 69:280-300. 